\section{Down's Anomaly}

2nd ed. By G. F. Smith and J. M. Berg. (Pp. ix + 348; 102 figures + 113 tables. £12.50) Edinburgh, London and New York: Churchill Livingstone. 1976.

A decade has passed since the first edition of Penrose and Smith's book on Down's Anomaly. In 1973, Lionel Penrose died. After Langdon Down, he had made the major contribution to our understanding of mongolism. The new edition pays tribute to both Penrose and Langdon Down and is written by George Smith, co-author of the first edition, and another Penrose devotee, Joe Berg. There have been many advances since the 1965 edition. Chromosome banding techniques have identified the chromosomes involved in translocation Down's. Studies on abortion material have indicated how frequently trisomy 21 occurs in first trimester abortions. The word amniocentesis does not appear in the index of the 1965 edition but is now a part of every woman's vocabulary. These changes are included in the new edition. Penrose campaigned for other changes. He would have preferred the word mongolism to have disappeared by now. It is, however, still deeply entrenched in everyday professional usage and is perhaps marginally better than 'Kalmuck idiocy' and the 'ill-finished' or 'unfinished' child.

With the birth incidence of Down's anomaly still as high as 1 in 660 live births, paediatricians, obstetricians, community physicians, and geneticists need a good source of reference on the subject. This second edition provides this. The chapter on vital statistics, cytology, and aetiology will answer all the practical questions about recurrence risks. The research worker or clinician cannot but be intrigued by the early onset of what seems to be Alzheimer's disease in mongols, the occurrence of severe infantile and transient forms of leukaemia, and the altered immune mechanism. The references are more than generous.

The only serious omission is in the section on 'social and educational considerations'. One looked for 'official' refutation of the still prevalent 'Madam, in my experience, your child will never learn to talk. Put him in an institution and forget you ever had him'. Despite this lack, Lionel Penrose would have been well satisfied with this second edition of his book.

\section{BARAITSER}

\section{Principles of Genetic Counselling}

By Edmond A. Murphy and Gary A. Chase. (Pp. 380; 14 Figures + 17 Tables. £13.75.) Year

Book Medical Publishers; Chicago; London: Lloyd-Luke. 1975.

Murphy and Chase have written an attractively individualistic account of genetic counselling. They are concerned with the philosophical and mathematical principles underlying counselling rather than with offering a detailed practical guide. The book will be hard reading for most medical practitioners and, indeed, for many medical geneticists, but rewarding for both. Even the experienced genetic counsellor will find himself taking a second look at some of the assumptions by which he practises the art.

A chapter on probability theory defines terms which are often used imprecisely. 'Likelihood', for example, the authors suggest, is best used for events that have already occurred. The chapter on counselling for Mendelian conditions is perhaps over-elaborated, but draws attention, for example, to the substantial affect on the estimate of risk for X-linked conditions provided by the unaffected males already present in the family. The chapter on ancillary methods sets out the important topic of how to combine information on risks from two independent sources-for example, from pedigree information and creatine kinase estimations in severe X-linked muscular dystrophy. A chapter on two locus systems is perhaps of mainly theoretical interest at present. The chapter on 'multilocal' systems, which follows the usual lines, does not appear to have seen the relation of the Edwards and Falconer models. The term 'multilocal' is proposed for a character which is under the control of several gene loci, reserving the term 'polygenic' for the situation in which the several gene loci are of about equal importance. The chapter on empirical risk counselling is sound in principle, but the text suggests some lack of experience of the realities of the more common malformations. The risk after several pregnancies affected by neural gene malformations is not 'comparatively small'. A table of risks for anencephaly by maternal age and social class has little relevance to genetic counselling in the usual situation, where parents have had one or more affected children or a parent himself is affected.

Genetic counselling is essentially a service to individual couples, but the authors are to be con- 